\title{
Perfil epidemiológico das lesões esportivas em atletas da seleção de badminton de
}

\section{Teresina-PI}

\author{
Epidemiological profile of sports injuries in athletes of the badminton selection of Teresina-PI \\ Perfil epidemiológico de lesiones deportivas en atletas de la selección de bádminton de Teresina-PI
}

Recebido: 07/05/2021 | Revisado: 16/05/2021 | Aceito: 21/05/2021 | Publicado: 22/05/2021

Demerval de Pinho Borges Netto

ORCID: https://orcid.org/0000-0002-9529-4130 Centro Universitário UNIFACID, Brasil

E-mail: demervalpinho15@outlook.com

Rayssa Maria de Araujo Carvalho

ORCID: https://orcid.org/0000-0002-2567-0209 Centro Universitário UNIFACID, Brasil

E-mail: rayssacarv@gmail.com

Neusa Barros Dantas Neta

ORCID: https://orcid.org/0000-0001-7127-1463 Centro Universitário UNIFACID, Brasil

E-mail: nbdn2@msn.com

Gabriela Dantas Carvalho

ORCID: https://orcid.org/0000-0002-9571-3323 Centro Universitário UNIFACID, Brasil E-mail: ftgabrieladantas@hotmail.com

Marcello de Alencar Silva

ORCID: https://orcid.org/0000-0001-9451-2979 Centro Universitário UNIFACID, Brasil E-mail: ft.alencar@gmail.com

\begin{abstract}
Resumo
O objetivo é investigar as principais lesões apresentadas pelos atletas de Badminton, como elas se manifestam e o impacto destas sobre o desempenho do atleta. Trata-se de uma pesquisa observacional, quantitativa, realizada em um Clube de esporte, no período de setembro a outubro de 2020, após a aprovação do Comitê de Ética do Centro Universitário UNIFACID, sob o número de parecer 4.276.525. Foram incluídos atletas de Badminton, com, no mínimo, 1 ano de prática no esporte. Os dados foram coletados através do questionário eletrônico (Google Forms). Os dados foram analisados usando SPSS 20.0, seguido do teste Kolmogorov-Smirnov Considerou-se significativo $\mathrm{p}<0,05$. Participaram 31 atletas, 51,6\% $(n=16)$ do gênero feminino e 48,4\% $(n=15)$ masculino, com média de idade de 17,9 anos $( \pm 2,6)$. Todos $(100 \%, n=31)$ com perfil competitivo, perfazendo uma média de 8,4 anos $( \pm 2,7)$ de tempo no esporte e 5,8 dias $( \pm 0,9)$ de ritmo de treino por semana. 41,9\% apresentaram dor, incomodo ou lesão, com comprometimento predominante no joelho $(38,7 \%, \mathrm{n}=12)$ e ombro $(12,9 \%, \mathrm{n}=4)$. $75,0 \%$ das lesões foram classificadas como leves e $100 \%$ com lesão grave $(\mathrm{p}=0,011)$, porém, sem associação da dor, incômodo ou lesão com gênero $(\mathrm{p}=0,722)$, decorrência da lesão $(\mathrm{p}=0,275)$, instabilidade articular $(\mathrm{p}=0,059)$ e interferência das lesões nas atividades diárias $(\mathrm{p}=0,262)$. Os atletas são veteranos dentro da modalidade e apresentam uma sequência intensa de treinos por semana, contudo, por ser um esporte de alta intensidade, sobrecarrega as articulações do joelho e ombro, culminando na instabilidade articular e na queda do rendimento esportivo.
\end{abstract}

Palavras-chave: Atletas; Lesões do esporte; Dor.

\begin{abstract}
The objective is to investigate the main origins by Badminton athletes, how they manifest themselves and the impact on the athlete's performance. This is an observational, quantitative research, carried out in a sports club, from September to October 2020, after approval by the Ethics Committee of the UNIFACID University Center, under the number of opinions 4,276,525. Badminton athletes were included, with at least 1 year of practice in the sport. The data were collected through the electronic questionnaire (Google Forms). The data were analyzed using SPSS 20.0, followed by the Kolmogorov-Smirnov test, $\mathrm{p}<0.05$ was considered significant. 31 athletes participated, $51.6 \%(\mathrm{n}=16)$ female and $48.4 \%(n=15)$ male, with a mean age of 17.9 years $( \pm 2.6)$. All $(100 \%, n=31)$ with a competitive profile, making an average of 8.4 years $( \pm 2.7)$ of time in sport and 5.8 days $( \pm 0.9)$ of training pace per week. $41.9 \%$ had pain, discomfort or injury, with predominant involvement in the knee $(38.7 \%, \mathrm{n}=12)$ and shoulder $(12.9 \%, \mathrm{n}=4)$. $75.0 \%$ of the injuries were classified as mild and $100 \%$ with severe injury $(\mathrm{p}=0.011)$, however, without association of pain, discomfort or injury with gender $(\mathrm{p}=0.722)$, due to the injury $(\mathrm{p}=0.275)$, instability articular $(\mathrm{p}=0.059)$ and
\end{abstract}


interference of lesions in daily activities $(\mathrm{p}=0.262)$. The athletes are veterans within the sport and have an intense sequence of training per week, however, as it is a high intensity sport, it overloads the knee and shoulder joints, culminating in joint instability and a drop in sports performance.

Keywords: Athletes; Sports injuries; Pain.

\section{Resumen}

El objetivo es investigar las principales lesiones que presentan los deportistas de Bádminton, cómo se manifiestan y su impacto en el rendimiento del deportista. Se trata de una investigación observacional, cuantitativa, realizada en un club deportivo, de septiembre a octubre de 2020, previa aprobación por parte del Comité de Ética del Centro Universitario UNIFACID, bajo el número de dictámenes 4.276.525. Se incluyeron atletas de bádminton, con al menos 1 año de práctica en el deporte. Los datos fueron recolectados a través del cuestionario electrónico (Formularios de Google). Los datos se analizaron con SPSS 20.0, seguido de la prueba de Kolmogorov-Smirnov, p <0.05 se consideró significativa. Participaron 31 deportistas, 51,6\% $(n=16)$ mujeres y 48,4\% $(n=15)$ hombres, con una edad media de 17,9 años $( \pm 2,6)$. Todos $(100 \%, n=31)$ con perfil competitivo, con un promedio de 8,4 años $( \pm 2,7)$ de tiempo en el deporte y 5,8 días $( \pm 0,9)$ de ritmo de entrenamiento por semana. El 41,9\% presentó dolor, malestar o lesión, con afectación predominante en rodilla $(38,7 \%, \mathrm{n}=12)$ y hombro $(12,9 \%, \mathrm{n}=4)$. El $75,0 \%$ de las lesiones se clasificaron como leves y el $100 \%$ con lesión grave $(\mathrm{p}=0,011)$, sin embargo, sin asociación de dolor, malestar o lesión con el sexo $(\mathrm{p}=0,722)$, debido a la lesión $(\mathrm{p}=0,275)$, inestabilidad articular $(\mathrm{p}=0,059)$ e interferencia de lesiones en las actividades diarias $(\mathrm{p}=0,262)$. Los deportistas son veteranos dentro del deporte y tienen una intensa secuencia de entrenamiento por semana, sin embargo, al ser un deporte de alta intensidad, sobrecarga las articulaciones de rodilla y hombro, culminando en inestabilidad articular y caída en el rendimiento deportivo.

Palabras clave: Deportistas; Lesiones deportivas; Dolor.

\section{Introduçãa}

O Badminton é um esporte que surgiu na Índia, denominado como poona, posteriormente, em 1870, disseminado na Europa pelos saldados Ingleses. Em 1934 foi fundada a Federação Internacional de Badminton com nove membros: Canadá, Dinamarca, Escócia, França, Holanda, Inglaterra, Nova Zelândia e País de Gales (Confederação Brasileira de Badminton, 2021). Sua partida lembra um jogo de voleibol e/ou tênis de quadra. No entanto, dispõe de regras diferentes, fazendo uso de peteca e raquete. As disputas acontecem de forma simples, em duplas e duplas mistas, em quadra dividida por uma rede, onde são feitos em três games de 21 pontos e seu principal objetivo é fazer a peteca cair na quadra do adversário (Sousa, 2017).

A Confederação Brasileira de Badminton classifica esse esporte como um jogo rápido e de agilidade (Sousa, 2017), em que a peteca alcança uma velocidade de $360 \mathrm{~km} / \mathrm{h}$ (Cabello \& Padial, 2002), necessitando de uma variedade de ações motoras num curto período (Cabello \& Padial, 2002; Cabello \& Gonzalez-Badillo, 2003). Segundo Cabello et al. (2004), o tempo total de uma partida individual de dois sets possui duração de 20 a 50 minutos, mas pode prolongar-se até três sets, podendo durar entre 70 a 75 minutos.

Considerando o perfil do esporte, o treinamento físico faz-se necessário para que o jogador desenvolva habilidades de equilíbrio, força e resistência, visando a prevenção de lesões musculares (LM) (Sousa, 2017). As LM apresentam alta incidência no meio esportivo, principalmente em atletas de alto rendimento, o que cursa na perda da capacidade física, afastamento de competições e insegurança quanto ao retorno no esporte (Miranda, 2018). Aproximadamente 42,5\% das lesões registradas no atletismo profissional acometem o tecido muscular, no qual em competições de alta performance, atingem $31 \%$ de todos os registros (Järvinen et al., 2005; Solaja et al., 2013).

As causas das lesões variam de acordo com o tipo de esporte, com as condições físicas do atleta e a intensidade de treinamento. Segundo Rahusen et al. (2004) a categorização dos mecanismos se baseia em conceitos mecânicos, respostas teciduais ou uma combinação dos dois, sendo intensificado quando a prática física é realizada de maneira extenuante e, ainda, inadvertida ou inapropriada (Pastre et al., 2005; Vargas et al., 2019).

As lesões esportivas são frequentemente subestimadas devido à ausência de notificação no universo esportivo, em especial, na modalidade esportiva de Badminton, que apesar de ser um esporte bem difundido pela Europa, ainda é pouco conhecido no Brasil. Contudo, é fundamental o conhecimento fisiopatológico do processo lesivo decorrente das modalidades 
esportivas, visando assistir o atleta na prevenção das lesões, adaptação dos treinos e na reabilitação daqueles já acometidos (Pastre et al., 2005). Dessa forma, o objetivo desse trabalho é investigar as principais lesões apresentadas pelos atletas de Badminton, como elas se manifestam e o impacto destas sobre o desempenho do atleta. Além disso, buscou-se verificar que fatores influenciaram na presença de dor, incomodo ou lesão e o nível de dor.

\section{Metodologia}

\section{Procedimentos Éticos}

O presente estudo foi realizado de acordo com as diretrizes para pesquisa preconizadas pela Resolução do Conselho Nacional de Saúde 466/12, sendo a mesma submetida à apreciação do Comitê de Ética em Pesquisa com Seres Humanos do Centro Universitário UNIFACID e aprovada sem restrições sob o número de parecer 4.276.525.

Os participantes da pesquisa assinaram o Termo de Consentimento Livre e Esclarecido (TCLE) e o Termo de Assentimento Livre e Esclarecido (TALE) por meio eletrônico ficando garantido o sigilo das informações por eles prestadas, comprovando que estavam cientes dos riscos, benefícios, procedimentos e objetivos da pesquisa.

\section{Desenho e participantes}

Trata-se de uma pesquisa observacional, com abordagem quantitativa, realizada em um Clube de esporte localizado em Teresina-PI, no período de setembro a outubro de 2020. A população acessível deste estudo foi composta atletas da seleção piauiense de Badminton.

Foram incluídos praticantes de Badminton sem distinção entre gêneros, com idade entre 15 e 25 anos, que tenham, no mínimo, 1 ano de prática no esporte. Foram excluídos praticantes que relataram problemas ortopédicos pregressos ao início da prática esportiva tais como: fraturas, entorses, distensões musculares e/ou tendíneas.

\section{Coleta de dados}

Os dados foram coletados por meio do questionário eletrônico, contendo 8 questões semiestruturadas, com tempo médio de reposta 30 minutos, norteadas a partir dos seguintes aspectos: perfil do atleta, possíveis lesões adquiridas na modalidade esportiva e as consequências no rendimento.

O questionário foi aplicado por um único pesquisador devidamente treinado e apto para aplicação do questionário, sendo utilizado a plataforma Google Forms.

\section{Organização e Análise dos Dados}

Os dados foram analisados usando Statistical Package for the Social Sciences (SPSS, Chicago, IL, EUA.), Versão 20.0. Foi realizada análise descritiva dos dados com os valores apresentados em frequências absolutas, porcentagens, média e desvio padrão, mediana, valores mínimo e máximo. Realizou-se o teste Kolmogorov-Smirnov para verificar o padrão de distribuição das variáveis quantitativas, verificou-se que o padrão de distribuição foi não normal. Assim, os testes nãoparamétricos selecionados foram: Mann-Whitney e Kruskal-Wallis. Também se realizou o Teste Exato de Fisher. Considerouse valor de $\mathrm{p}<0,05$ como significativo. Os dados foram expressos em tabelas.

\section{Resultados}

Participaram do estudo 31 atletas, onde 51,6\% ( $n=16)$ eram do gênero feminino e 48,4\% (n=15) do gênero masculino. Todos os atletas $(100 \%, \mathrm{n}=31)$ eram de Teresina e possuíam perfil competitivo, 41,9\% apresentaram dor, incomodo ou lesão em treinos. A área do corpo que já foi mais afetada por dor foi o joelho $(38,7 \%, n=12)$ e ombro $(12,9 \%, n=4)$. A média de idade dos participantes foi 17,9 anos $( \pm 2,6)$. Os atletas treinavam por uma média de 3,5 horas $( \pm 1,3)$, possuíam média 
8,4 anos $( \pm 2,7)$ de tempo no esporte, treinavam em média 5,8 dias $( \pm 0,9)$ na semana e já apresentaram um valor médio de dor de $2,1( \pm 2,6)$, conforme apresentado na Tabela 1 .

Tabela 1: Análise descritiva do perfil sociodemográfico e de treinamento de atletas do Badminton de Teresina-PI ( $\mathrm{n}=31$ ).

n $\%$

\section{Gênero}

Feminino 16 51,6

Masculino

\section{Naturalidade}

Teresina

\section{Perfil do atleta}

Competitivo

Intermediário

Iniciante

\section{Dor, incomodo ou lesão em treinos}

Sim

Não

Área do corpo mais acometida pela dor

Joelho

Tornozelo

2

6,5

Quadril

2

6,5

Punho

1

Ombro 4

Mãos

3

9,7

Costas 4

Lombar 1 3,2

Ombro

1

Virilha

00,0

0,0

$\%$

,9

\begin{tabular}{lcc}
\hline & Média (desvio padrão) & Mediana (valor mínimo e máximo \\
\hline Idade & $17,9(2,6)$ & $17,0(15-25)$ \\
Tempo no esporte & $8,4(2,7)$ & $8,0(2-13)$ \\
Horas de treino & $3,5(1,3)$ & $4,0(1-7)$ \\
Dias na semana & $5,8(0,9)$ & $6,0(2-7)$ \\
Nível da dor & $2,1(2,6)$ & $0(0-7)$ \\
\hline
\end{tabular}

Legenda: $n=$ Número de participantes; \%= percentual. Fonte: Autores (2020).

A Tabela 2 apresenta a percepção dos atletas sobre o grau da lesão e como ela impacta sobre seu rendimento esportivo e atividades diárias, mostrando que houve predomínio de lesão com grau leve e grave. 75,0\% das lesões leves acusaram não 
sentir dor ou incômodo na área acometida, enquanto $100 \%$ dos atletas com lesão grave referiram dor ou incômodo (p=0,011). Não houve associação da dor, incômodo ou lesão com gênero ( $\mathrm{p}=0,722)$, decorrência da lesão $(\mathrm{p}=0,275)$, instabilidade articular $(\mathrm{p}=0,059)$ e interferência das lesões nas atividades diárias $(\mathrm{p}=0,262)$. Quanto ao rendimento esportivo nos últimos 6 meses, todos os atletas que relataram queda no rendimento, 100,0\% apresentaram associação com dor ou incômodo ( $\mathrm{p}=0,023$ ).

Tabela 2: Associação entre presença de dor, incomodo ou lesão com gênero, decorrência da lesão, grau de lesão, instabilidade articular e interferência das lesões nas atividades diárias de atletas do Badminton de Teresina-PI.

\begin{tabular}{|c|c|c|c|c|}
\hline & & \multicolumn{2}{|c|}{ Dor, incomodo ou lesão } & \multirow{2}{*}{ Valor de $\mathrm{p}$} \\
\hline & & $\operatorname{sim}$ & não & \\
\hline \multirow{4}{*}{ Gênero } & \multirow{2}{*}{ Feminino } & $6 a$ & $10_{a}$ & 0,722 \\
\hline & & $37,5 \%$ & $62,5 \%$ & \\
\hline & \multirow{2}{*}{ Masculino } & $7 \mathrm{a}$ & $8 \mathrm{a}$ & \\
\hline & & $46,7 \%$ & $53,3 \%$ & \\
\hline \multirow{4}{*}{ Lesão decorrente } & \multirow{2}{*}{ treino } & $10 \mathrm{a}$ & $10_{\mathrm{a}}$ & 0,275 \\
\hline & & $50,0 \%$ & $50,0 \%$ & \\
\hline & \multirow{2}{*}{ competição } & $3 \mathrm{a}$ & $8 \mathrm{a}$ & \\
\hline & & $27,3 \%$ & $72,7 \%$ & \\
\hline \multirow{6}{*}{ Grau da lesão } & \multirow{2}{*}{ leve } & $4 a$ & $12 \mathrm{~b}$ & $0,011 * * *$ \\
\hline & & $25,0 \%$ & $75,0 \%$ & \\
\hline & \multirow{2}{*}{ moderado } & $5 \mathrm{a}$ & $3 \mathrm{a}$ & \\
\hline & & $62,5 \%$ & $37,5 \%$ & \\
\hline & \multirow{2}{*}{ grave } & $4 a$ & $\mathrm{Ob}_{\mathrm{b}}$ & \\
\hline & & $100,0 \%$ & $0,0 \%$ & \\
\hline \multirow{4}{*}{ Instabilidade articular } & \multirow{2}{*}{$\operatorname{sim}$} & $5 \mathrm{a}$ & $1^{\mathrm{a}}$ & 0,059 \\
\hline & & $83,3 \%$ & $16,7 \%$ & \\
\hline & \multirow{2}{*}{ não } & $8 \mathrm{a}$ & $17 \mathrm{a}$ & \\
\hline & & $32,0 \%$ & $68,0 \%$ & \\
\hline \multirow{6}{*}{$\begin{array}{c}\text { Interferência das lesões nas } \\
\text { atividades diárias }\end{array}$} & \multirow{2}{*}{$\begin{array}{c}\text { interfere } \\
\text { diretamente }\end{array}$} & $0 \mathrm{a}$ & $1 \mathrm{a}$ & 0,262 \\
\hline & & $0,0 \%$ & $100,0 \%$ & \\
\hline & \multirow{2}{*}{$\begin{array}{l}\text { interfere em } \\
\text { parte }\end{array}$} & $7 \mathrm{a}$ & $5 \mathrm{a}$ & \\
\hline & & $58,3 \%$ & $41,7 \%$ & \\
\hline & \multirow{2}{*}{ não interfere } & $6 a$ & $12 \mathrm{a}$ & \\
\hline & & $33,3 \%$ & $66,7 \%$ & \\
\hline \multirow{4}{*}{$\begin{array}{c}\text { Queda do rendimento esportivo } \\
\text { últimos } 6 \text { meses }\end{array}$} & \multirow{2}{*}{$\operatorname{sim}$} & $4 a$ & $\mathrm{Ob}_{\mathrm{b}}$ & $0,023 * *$ \\
\hline & & $100,0 \%$ & $0,0 \%$ & \\
\hline & \multirow{2}{*}{ não } & $9 \mathrm{a}$ & $18 \mathrm{~b}$ & \\
\hline & & $33,3 \%$ & $66,7 \%$ & \\
\hline \multirow{2}{*}{\multicolumn{2}{|c|}{ Total }} & 13 & 18 & \\
\hline & & $41,9 \%$ & $58,1 \%$ & \\
\hline
\end{tabular}

Legenda: *p valor estatisticamente significante (Teste Exato de Fisher). Letras diferentes indicam sentido da associação estatística. Fonte: Autores (2020). 
Ao associar a idade dos atletas ao seu nível de preparo esportivo e o desencadeamento dor, incômodo ou lesão, a Tabela 3 mostra que os atletas que relataram dor, incômodo ou lesão tiveram maior mediana de idade $(18$ anos, p=0,705) e tempo no esporte (11 anos, $\mathrm{p}=0,135)$, mas sem significância estatística. Também não houve associação significativa entre presença de dor, incômodo ou lesão com valores medianos de horas de treino $(\mathrm{p}=0,373)$ e quantidade de dias na semana em que há o treinamento $(\mathrm{p}=0,859)$.

Tabela 3: Distribuição da mediana de idade, tempo no esporte, quantas horas de treino e quantos dias na semana pela presença de dor, incomodo ou lesão em atletas do Badminton de Teresina-PI ( $\mathrm{n}=31)$.

\begin{tabular}{cccccc}
\hline & Dor, incomodo ou lesão & Idade & $\begin{array}{c}\text { Tempo no } \\
\text { esporte }\end{array}$ & Horas de treino & Quantos dias na semana \\
\hline Sim & $\begin{array}{c}\text { Mediana (valor mínimo }- \\
\text { máximo) }\end{array}$ & $18,0(15-25)$ & $11,0(5-13)$ & $3,0(1-7)$ & $6,0(2-7)$ \\
\hline Não & $\begin{array}{c}\text { Mediana (valor mínimo }- \\
\text { máximo) }\end{array}$ & $17,0(15-21)$ & $8,0(2-12)$ & $4,0(2-5)$ & $6,0(5-7)$ \\
\hline Total & $\begin{array}{c}\text { Mediana (valor mínimo - } \\
\text { máximo) }\end{array}$ & $17,0(15-25)$ & $8,0(2-13)$ & $4,0(1-7)$ & $6,0(2-7)$ \\
\hline & Valor de p & 0,705 & 0,135 & 0,373 & 0,859 \\
\hline
\end{tabular}

Legenda: *p valor estatisticamente significante (Teste Mann-Whitney). Fonte: Autores (2020).

Quanto ao impacto causado no rendimento dos atletas nas atividades esportivas após as lesões quem tinha grau de lesão grave $(5,5 ; \mathrm{p}=0,027)$, apresentava instabilidade articular $(3,5 ; \mathrm{p}=0,014)$ e queda no rendimento esportivo nos últimos 6 meses $(5,5 ; \mathrm{p}=0,047)$ apresentou maior mediana de nível de dor (Tabela 4).

Tabela 4: Distribuição da mediana do nível de dor pelo gênero, perfil do atleta, decorrência da lesão, grau de lesão, instabilidade articular, interferência das lesões em atividade diárias e queda no rendimento esportivo nos últimos 6 meses de atletas do Badminton de Teresina-PI ( $\mathrm{n}=31)$.

\begin{tabular}{|c|c|c|c|}
\hline & Mediana & Valor mínimo-máximo & Valor de $\mathrm{p}$ \\
\hline Gênero & & & 0,716 \\
\hline Feminino & 0 & $0-6$ & \\
\hline Masculino & 1 & $0-7$ & \\
\hline Perfil do atleta & & & 1,000 \\
\hline Competitivo & 0 & $0-7$ & \\
\hline Intermediário & 0 & & \\
\hline Iniciante & 0 & & \\
\hline Lesão decorrente & & & 0,325 \\
\hline Treino & 1,5 & $0-6$ & \\
\hline Competição & 0 & $0-7$ & \\
\hline Grau de lesão & & & $0,027 * *$ \\
\hline Leve & 0 & $0-5$ & \\
\hline Moderado & 3 & $0-7$ & \\
\hline Grave & 5,5 & $5-6$ & \\
\hline
\end{tabular}




\section{Instabilidade articular}

$\operatorname{Sim}$

Não

\section{Interferência das lesões em} atividade diárias

Interfere diretamente

Interfere em parte

Não interfere

Queda no rendimento esportivo nos últimos 6 meses

Sim

Não
3,5

0

0

3,5

0

$0-7$

0

$0-6$

$0-7$

$0,047 * *$

0

$0-6$

\section{Discussão}

Segundo a Rede Nacional do Esporte (2019), o município de Teresina-PI é conhecido como a capital da peteca e da raquete. Celeiro da modalidade de Badminton, Teresina desenvolve o esporte desde 2009, atualmente contando com uma ampla estrutura física localizada na Universidade Federal do Piauí, onde possibilita o desenvolvimento dos treinos e preparação dos atletas para as competições. Segundo o presidente da Confederação Brasileira de Badminton, esporte que exige muita técnica, uma vez que a peteca é leve, com peso de cerca de 5 gramas, podendo o atleta realizar movimentos de alta intensidade que culminam em lesões.

Segundo a Confederação Brasileira de Badminton (2005) as lesões esportivas são provocadas por métodos inadequados de treinamento, por alterações estruturais que sobrecarregam mais determinadas partes do corpo do que outras e pela fraqueza muscular dos tendões e dos ligamentos. Muitas dessas lesões são causadas pelo desgaste crônico e por lacerações, que são decorrentes de movimentos repetitivos que afetam os tecidos suscetíveis.

O Badminton apesar de ser um esporte bem difundido nos continentes asiático e europeu, ainda é considerado uma modalidade esportiva recente no Brasil. Segundo Fontes et al. (2014) existe uma carência na literatura referente ao tema, o que dificulta o estabelecimento de uma comparação entre os dados. Entretanto, diante do crescimento da modalidade esportiva, estudos referentes à temática tornam-se relevantes, pois oferecem orientações quanto ao conhecimento do esporte, as possíveis lesões desencadeadas pela sua prática, contribuindo efetivamente no aperfeiçoamento da técnica e na prevenção de agravos.

O Badminton pode ser incluído na lista de modalidades de esportes que podem promover aumento do risco de lesões e alterações posturais, dado os momentos realizados e a velocidade da execução destes (Oliveira et al., 2004). Segundo Loureiro et al. (2012) as exigências posturais determinadas pelos movimentos executados durante esportes com raquete envolvem uma sequência de ativações neuromusculares que abrangem a rotação do tronco, a ativação da pelve, a rotação interna do ombro, a extensão do cotovelo e flexão do punho.

Rodriguez et al. (2020) correlaciona a incidência de lesões esportivas com a idade e gênero. Ribeiro et al. (2003) descrevem que o organismo jovem encontra-se em fase de desenvolvimento, sendo mais suscetível a sobrecargas externas e, consequentemente, a lesões osteomioarticulares. Baseado nisso, ao analisar o perfil dos atletas de Badminton, observou-se uma maior prevalência de indivíduos entre 15 a 17 anos e do gênero feminino. O gênero feminino esteja mais propenso a lesões esportivas dada aos inúmeros fatores anátomo-fisiológicos, tais como: resposta neuromuscular, diferenças morfológicas e hormonais. Segundo Gayardo et al. (2012), mulheres tem um tempo de ativação neuromuscular superior ao dos homens, 
podendo afetar a dinâmica articular. Outro fator importante é o alargamento do quadril que conduz ao aumento do ângulo Q e os joelhos em genu valgum, favorecendo disfunções patelo-femorais. Além do fator hormonal dado pela presença da relaxina, que promove o relaxamento excessivo das estruturas ligamentares, bem como o estrógeno que atua favorecendo na resposta álgica e no atraso da resposta motora (Baldon et al., 2011).

O Badminton é considerado um dos esportes mais rápidos do mundo, é uma atividade física de alto gasto energético, sendo assim, o jogador necessita de agilidade para realizar manobras de defesa em tempo ágil, o que contribui para uma série de lesões. Segundo Almeida et al. (2009) o alongamento muscular antes do treino com o objetivo de prevenir lesão. Fatores como idade, tipo de alongamento, frequência de treino, duração e intensidade devem ser considerados na prevenção.

Ao ser analisado as estruturas anatômicas mais afetadas na prática do Badminton, observa-se maior prevalência a articulação do joelho e ombro. Segundo Machado (2004) durante movimentos de alto impacto, torna-se essencial a coordenação e ativação sincronizada da flexão e extensão do joelho, de forma a aumentar a reação contra chão. A força transferida do joelho segue para o quadril e o tronco, assim, ao angular seu tronco o atleta é capaz de criar diversas angulações musculares, sobrecarregando sua articulação. O tronco dá velocidade ao braço, que, por sua vez, gira em torno do ombro, estendendo o cotovelo e atuando sobre o punho.

Oelke (2007) ao analisar as alterações posturais em atletas da Seleção Brasileira de Badminton, na categoria adulta, analisou seis atletas do gênero feminino e dois do gênero masculino, na faixa etária entre 19 e 27 anos de idade, aos quais apresentaram aumento da rotação interna do ombro, do ângulo $Q$ e da distância entre o retropé direito e esquerdo, o que favorecem o desencadeamento de lesões na articulação do ombro e de membros inferiores.

As lesões em membro inferior são um fator de grande significância em jogadores de Badminton, com destaque para a lesão de tornozelo que indica uma porcentagem de $(63,3 \%)$ contribuindo para o afastamento e queda no rendimento em treinos e competições esportivas. A maior frequência de lesões ocorre devido à falta de habilidade e preparo no esporte de Badminton (Kang \& Ramaligan, 2019). Segundo a Federação Portuguesa de Badminton (2010) a entorse da tibiotársica é uma das lesões mais presentes em atleta de Badminton, na qual pode ocorrer quando pisa o pé do parceiro, quando posiciona mal o pé na receção ao solo após um salto ou num simples deslocamento lateral.

Santos \& Greguol (2017) descrevem que as lesões esportivas estão associadas ao treinamento inadequado, falta de assistência e falta de estrutura adequada para o treino. Segundo os dados coletados, observa-se predominância de lesões durante os treinos, porém com baixa reincidência e instabilidade articular, sugerindo lesões de baixa intensidade, uma vez que os atletas relataram não haver afastamento significante dos treinos, sem queda de rendimento e com baixo impacto na qualidade de vida dos envolvidos. Acredita-se que a prevalência de lesões no treinamento seja decorrente do processo de adaptação que os atletas passam até estarem aptos a participarem dos campeonatos. Os atletas participam ativamente de competições e torneios nacionais e internacionais. O time de Badminton do município de Teresina tornou-se referência nacional pela qualidade técnica e inúmeras vitórias adquiridas. O time conta com acompanhamento do Fisioterapeuta à disposição em treinos e jogos, principalmente nos grandes eventos, faz-se necessário no preparo físico dos atletas, na prevenção e recuperação de lesões.

\section{Conclusão}

Os atletas são veteranos dentro da modalidade e apresentam uma sequência intensa de treinos por semana o que favorece a adaptação do organismo ao esporte, contudo, por ser um esporte de alta intensidade, com movimentos rápidos, sobrecarregando, em especial, as articulações do joelho e ombro. A presença de dor foi influenciada pelo tipo de lesão grave, culminando na instabilidade articular e na queda do rendimento esportivo nos últimos 6 meses. 


\section{Referências}

Almeida, S. L., Silva, L; I., \& Anacleto, C. M. G. (2019). Revisão integrativa sobre lesões e psicologia do esporte. Revista Científica UMG, 4(3), http://seer.umc.br/index.php/revistaumc/article/view/917/700.

Aragão, M., Lima, A., Mota, D., Pessoa, D., \& Leal, S. (2019). Efeitos do treinamento com a realidade virtual (Kinect Sports®) sobre os níveis de equilíbrio e agilidade em jogadores de badminton: estudo clínico controlado e randomizado. Revista Saúde, 45(3). doi:https://doi.org/10.5902/2236583439452.

Araujo, K. M., Baeza, M. R., Zalada, B. R. S., Alves, R. B. P., \& Mattos, A. C. (2015). Lesões em praticantes amadores de corrida. Revista Brasileira de Ortopedia, 50 (5), 537-540. https://doi.org/10.1016/j.rbo.2015.04.003.

Baldon, R. M., Lobato, D. F. M., Carvalho, L. P., Wun, P. Y. L., \& Serrão, F. V. (2011). Diferenças biomecânicas entre os gêneros e sua importância nas lesões do joelho. Fisioterapia em Movimento, 24(1), 157-166. https://doi.org/10.1590/S0103-51502011000100018.

Breno Barros. Rede Nacional do Esporte (2019) Treinos da Seleção Brasileira de Badminton no Piauí unem alto rendimento e pesquisa científica, rededoesporte. gov.br

Cabello, D., \& Padial, P. (2002). Análisis de los parámetros temporales en um partido de Bádminton. Motricidad: Revista de Ciencias de la Actividad Física y del Deporte, n. 9,101-117.

Cabello, D., \& Gonzalez-Badillo, J. J. (2003). Analysis of the characteristics of competitive badminton. British Journal of Sports Medicine. v.37,62-66. http://doi: $10.1136 /$ bjsm.37.1.62.

Cabello, D., Prada, A., Sanchez, A., Sicilia, A., \& Corral, F. (2004). Analisis informatizado del juego en jugadores de bádminton de elite mundial: Computerized analysis of game in tope-class badminton players. Revista Cultura, Ciência e Esporte,1(1), 25-31. http://dx.doi.org/10.12800/ccd.v1i1.12.

Confederação Brasileira de Badminton (2005). Lesões no esporte: um mal que pode ser evitado.http://www.badminton.org.br/noticia/1169/-lesoes-noesporte-um-mal-que-pode-ser-evitado

Federação Portuguesa de Badminton (2010) Lesões mais comuns no Badminton. https://fpbadminton.pt/wp-content/uploads/Leso\%cc\%83es-mais-comuns.pdf

Fontes, T. T., Chiminazzo, J. G. C., Dobránszky, I. de A.; \& Moraes, A. M. (2014). Análise da quantificação das ações motoras e da estrutura temporal no badminton. Revista Brasileira De Prescrição E Fisiologia Do Exercício, 8(50),782-786. http://www.rbpfex.com.br/index.php/rbpfex/article/view/702.

Gayardo, A., Matana, S. B., \& Silva, M. R. (2012). Prevalência de lesões em atletas do futsal feminino brasileiro: um estudo retrospectivo. Revista Brasileira de Medicina do Esporte, 18(3), 186-189. https://doi.org/10.1590/S1517-86922012000300010.

Järvinen, T. A., Järvinen, T. L., Kääriäinen, M., Kalimo, H., \& Järvinen, M. (2005). Muscle injuries: biology and treatment. The American journal of sports medicine, 33(5), 745-764. https://doi.org/10.1177/0363546505274714.

Kang, A. L., \& Ramalingam, V. (2018). Risk factors for lower extremity injuries in young badminton players. Revista Scientia Medica,28(2). https://doi.org/10.15448/1980-6108.2018.2.28939.

Loureiro JR., L. F. B., \& Freitas, P. B. (2012) Influência do nível de desempenho de jogadores de badminton nos aspectos neuromotores durante uma tarefa de apontar um alvo. Revista Brasileira Medicina do Esporte .18(3) ,203-207. http://dx.doi.org/10.1590/S1517-86922012000300014.

Machado, E. S. (2004) A transmissão da vibração no cotovelo após o impacto da raquete na bola e consequências pelo mau uso da técnica e do material: aspectos teóricos e pesquisa experimental para a medição das acelerações do cotovelo no impacto da bola na raquete na prática do saque chapado. $116 \mathrm{f}$. Trabalho de Conclusão de Curso de Educação Física. PUC/RS; Porto Alegre.

Miranda, T. A. R., Lemes, R. I., Castrillón, M. I. C., Vanderlei, M. F., Linares, N. S., Christofaro, D. G. D., Pastre, M. C., \& Netto Júnior, J. (2018). Lesões musculares em atletas do sexo masculino atendidos no Centro de Estudos e Atendimento em Fisioterapia de Presidente Prudente - SP. Revista Brasileira de Ciências do Esporte, 40(1), 70-76. https://doi.org/10.1016/j.rbce.2018.01.007

Oelke, P. (2007) Avaliação Postural em Atletas da Seleção Brasileira de Badminton. Revista Brasileira Medicina do Esporte. 18(6), 76-81.

Oliveira, M. M. M., Lourenço, M. R. A., \& Teixeira, D. C. (2015) Incidências de lesões nas equipes de ginástica rítmica da Unopar. Revista Unopar Cient Biol Saúde, 5/6 (1),29-40. https://doi.org/10.17921/2447-8938.2003v0n0p\%25p.

Praste, M. C., Filho, C. G., Monteiro, L. H., Júnior, N. J., \& Padovani, R. C. (2005) Lesões desportivas na elite do atletismo brasileiro: estudo a partir de morbidade referida. Revista Brasileira Medicina do Esporte, 11(1), 43-47. https://doi.org/10.1590/\$1517-86922005000100005.

Rahusen, F. T., Weinhold, P. S., \& Almekinders, L. C. (2004). Nonsteroidal anti-inflammatory drugs and acetaminophen in the treatment of an acute muscle injury. The American journal of sports medicine, 32(8), 1856-1859. https://doi.org/10.1177/0363546504266069.

Ribeiro, C. Z. P., Akashi, P. M. H., Sacco, I. C. N., \& Pedrinelli, A. (2003) Relação entre alterações posturais e lesões do aparelho locomotor em atletas de futsal. Revista Brasileira Medicina do Esporte, 9(2),98-103. https://doi.org/10.1590/S1517-86922003000200005.

Rodrigues, M. A. B., Guimarães, N. R., Nogueira, A. Z. H. J., \& Donner, D. (2017) Atuação da Fisioterapia na Prevenção de Lesões em Atletas. Revista Revista Brasileira De Prescrição E Fisiologia Do Exercício, 11(4), 101-110.

Rodríguez, M. A., Nogueron, G. G., Manrique, C. D., \& Avila, O. B. A. (2020) Incidence of injuries among amateur badminton players: A cross-sectional study. Revista Medicine, 99(18). https://doi: 10.1097/MD.0000000000019785.

Santos, M. A., \& Greguol, M. (2017) Prevalência de lesões em atletas jovens. Semina: Ciências Biológicas e da Saúde, 37(2), 115-124. http://dx.doi.org/10.5433/1679-0367.2016v37n2p115. 
Research, Society and Development, v. 10, n. 6, e46910615865, 2021

(CC BY 4.0) | ISSN 2525-3409 | DOI: http://dx.doi.org/10.33448/rsd-v10i6.15865

Solaja, Anita \& Solaja, Milan \& Milankov, Miroslav. (2013). Injuries in elite athletes in the Olympic period from 2008 to 2012. Medicinski pregled. 66(1112). 483-490. https://doi.org/10.2298/MPNS1312483S

Souza, A. A., Raasch, H. N. R., \& Maria, L. A. (2017) Badminton: Um diferencial nas aulas de educação física. Estudos do Movimento Humano. Revista ACTA Brasileira do Movimento Humano, 7(3), 28-38. http://www.periodicos.ulbra.br/index.php/actabrasileira/issue/view/248

Vargas, L. M., Vargas, T. M., \& Zaremba, C. M. (2019). Prevalência de lesões osteomusculares e fatores associados em atletas participantes dos Jogos Universitários Brasileiros 2017. RBPFEX-Revista Brasileira De Prescrição E Fisiologia Do Exercício, 13(82), 329342.http://www.rbpfex.com.br/index.php/rbpfex/article/view/1717. 